\title{
Interpretación del aumento de peso tras un suplemento nutricional en diálisis peritoneal: a propósito de dos
} casos

\author{
Francisca Muelas Ortega, Sagrario Jiménez Jiménez, Pilar Segura Torres*, Francisco José Borrego \\ Utiel*, José Manuel Gil Cunquero*, Antonio Liébana Cañada*
}

Enfermera, *Facultativo Especialista de Nefrología. Unidad de Diálisis Peritoneal. Servicio de Nefrología. Complejo Hospitalario de Jaén

\section{Introducción}

La malnutrición es frecuente en pacientes con insuficiencia renal crónica en diálisis estimándose que afecta al $18-78 \%$ de los que están en diálisis peritoneal (DP)․․ En los últimos años multitud de trabajos han puesto de manifiesto que la malnutrición en diálisis constituye un factor predictor de mayor mortalidad ${ }^{2}$ y de mayor morbilidad, relacionándose también con una mayor frecuentación hospitalaria y mayor duración de los ingresos hospitalarios ${ }^{3}$.

Existen numerosos factores implicados en la malnutrición en DP como la escasa ingesta oral favorecida por múltiples factores como edad, pérdida de función renal residual y la diálisis inadecuada ${ }^{1}$; enfermedades crónicas asociadas; baja eficacia de la diálisis ${ }^{4}$; el estado inflamatorio que favorece la anorexia y que genera catabolismo proteico y la pérdida de nutrientes por el líquido dializado que aumenta cuando existe peritonitis ${ }^{1}$.

Debido a la alta prevalencia de malnutrición en DP, se han utilizado suplementos proteicos en pacientes con poca masa muscular ${ }^{1}$ para intentar incrementar la ingesta proteica y también los niveles de albúmina y de masa magra ${ }^{5}$.

Aunque existen varios métodos para valorar el estado nutricional, no disponemos de un gold standard. En este

Correspondencia:

Francisca Muelas Ortega

C/ Del Real Jáen, 1. $5^{\circ}$ F. 23003. Jaén

e-mail: atmosferacero@telefonica.net sentido puede ayudarnos el análisis de bioimpedancia (BIA) que nos permite analizar por separado el estado de nutrición y el de hidratación. Fein y su grupo en el $2002^{6}$ y Mushnick y su grupo en el $2003^{7}$ concluyen que la BIA es un método útil para valorar el estado nutricional en pacientes en DP.

El aumento de peso de un paciente en diálisis peritoneal que toma un suplemento nutricional puede tener varias interpretaciones no excluyentes entre sí. El aumento de peso podría deberse a un aumento de líquido o bien a una mejora del estado nutricional 0 incluso a ambos a la vez. Es importante determinar a que es debido el aumento de peso porque conlleva una actitud terapéutica diferente.

\section{Ojetivo}

Interpretar el incremento del peso en diálisis peritoneal tras un suplemento nutricional.

\section{Pacientes y métodos}

Seleccionamos dos pacientes que llevaban más de 3 meses en diálisis peritoneal y que tomaron un suplemento nutricional ( 2 bricks de $\mathrm{NEPRO}^{\circledR}$ al día) durante 2 meses y ganaron peso con respecto a la revisión previa.

Para interpretar el aumento de peso utilizaremos los siguientes parámetros:

- Peso en la revisión previa y en la revisión actual.

- Medidas antropométricas: Los parámetros a medir son circunferencia de la pantorrilla, del muslo y del 
brazo. Pliegue de grasa subcutánea de la pantorrilla, del muslo, bicipital y tricipital para lo cual se ha empleado la metodología recomendada por Alastrué ${ }^{8}$. El brazo utilizado será el que no tenga fístula y la pierna del mismo lado. Para la medición de los pliegues se empleará un lipocalibrador tipo LANGE, se realizaron tres mediciones y se anotó la media de las tres.

- Volumen de diuresis.

- Volumen de ultrafiltración total en 24 horas.

- Tensión arterial.

- Número de hipotensores.

- Albúmina.

- Exploración física: valorar presencia de edemas en piernas.

- Análisis de impedancia bioeléctrica (BIA) con BIA 101 Anniversary (Akern Bioresearch), tras 15 minutos en reposo y postdrenaje de líquido peritoneal. Se calculó agua corporal total y masa grasa.

\section{Resultados}

Caso clínico 1: Varón de 57 años con insuficiencia renal crónica secundaria a glomerulonefritis crónica en diálisis peritoneal desde hace unos 2 años en DPCA con 4 cambios de 2 litros sin hipertónicos (Gambro). Tras el suplemento aumentó el peso en 2,8 kg con respecto al peso de la revisión previa (revisión basal $77,2 \mathrm{~kg}$ y a los 2 meses $80 \mathrm{~kg}$ ). Aunque la tensión arterial aumentó un poco, pero estaba bien controlada pasando de $122 / 61 \mathrm{mmHg}$ a $130 / 90 \mathrm{mmHg}$ pero se le había quitado un hipotensor pasando de tomar tres a dos. Aumentó un poco el volumen de ultrafiltración en $24 \mathrm{~h}$ de $70 \mathrm{ml}$ a $130 \mathrm{ml}$ permaneciendo el volumen de diuresis igual en $1000 \mathrm{ml} /$ día. Los niveles de albúmina aumentaron de 3,1 g/dl a 3,4 g/dl. La exploración física en cuanto a valoración de edemas no cambió, no presentando edemas en ningún momento. Con respecto al resto de exploración nutricional encontramos que aumentaron la circunferencia de la pantorrilla de 33 $\mathrm{cm}$ a $36 \mathrm{~cm}$ y del brazo de $26 \mathrm{~cm}$ a $28,5 \mathrm{~cm}$, y la del muslo no cambió quedando en $42 \mathrm{~cm}$ (Tabla I), también aumentaron todos los pliegues grasos de tal forma que el de la pantorrilla pasó de $15,5 \mathrm{~mm}$ a $16,2 \mathrm{~mm}$, del muslo de $15,7 \mathrm{~mm}$ a 18,2 $\mathrm{mm}$, el bicipital de $6,6 \mathrm{~mm}$ a $8 \mathrm{~mm}$ y el tricipital de 7,5 mm a 8,2 $\mathrm{mm}$ (Tabla II). Finalmente el análisis de bioimpedancia nos indica que no ha aumentado el agua corporal total siendo el basal de 45,2 litros y final de 45,1 litros, con aumento importante de la masa grasa del paciente pasando de $15,7 \mathrm{~kg}$ a $18,3 \mathrm{~kg}$ (Tabla III).

\begin{tabular}{|l|c|c|c|c|c|c|}
\hline & C panto B & C panto F & C muslo B & C muslo F & C brazo B & C brazo F \\
\hline Caso 1 & $33 \mathrm{~cm}$ & $36 \mathrm{~cm}$ & $42 \mathrm{~cm}$ & $42 \mathrm{~cm}$ & $26 \mathrm{~cm}$ & $28,5 \mathrm{~cm}$ \\
\hline Caso 2 & $33 \mathrm{~cm}$ & $35 \mathrm{~cm}$ & $47,5 \mathrm{~cm}$ & $47 \mathrm{~cm}$ & $32,5 \mathrm{~cm}$ & $36,5 \mathrm{~cm}$ \\
\hline
\end{tabular}

B: basal F: final. C: circunferencia. Panto: pantorrilla

Tabla 1. Evolución de las circunferencias de pantorrilla, muslo y brazo

\begin{tabular}{|c|c|c|c|c|c|c|c|c|}
\hline & P pant Basal & P pant Final & P musl Basal & P musl Final & P bicip Basal & P bicip Final & P trici Basal & P trici Final \\
\hline Caso 1 & $15,5 \mathrm{~mm}$ & $16,2 \mathrm{~mm}$ & $15,7 \mathrm{~mm}$ & $18,2 \mathrm{~mm}$ & $6,6 \mathrm{~mm}$ & $8 \mathrm{~mm}$ & $7,5 \mathrm{~mm}$ & $8,2 \mathrm{~mm}$ \\
\hline Caso 2 & $19 \mathrm{~mm}$ & $22,5 \mathrm{~mm}$ & $37 \mathrm{~mm}$ & $29,7 \mathrm{~mm}$ & $17 \mathrm{~mm}$ & $12 \mathrm{~mm}$ & $28 \mathrm{~mm}$ & $20 \mathrm{~mm}$ \\
\hline
\end{tabular}

P: pliegue. Pant: pantorrilla. Musl: muslo. Bicip: bicipital. Trici: tricipital

Tabla 2. Evolución de los pliegues grasos en pantorrilla, muslo y brazo

\begin{tabular}{|l|c|c|c|c|}
\hline & Agua corporal B & Agua corporal F & Masa grasa B & Masa grasa F \\
\hline Caso 1 & 45,2 litros & 45,1 litros & $15,7 \mathrm{~kg}$ & $18,3 \mathrm{~kg}$ \\
\hline Caso 2 & 31,9 litros & 33,6 litros & $38,7 \mathrm{~kg}$ & $39,1 \mathrm{~kg}$ \\
\hline
\end{tabular}

B: basal F: final

Tabla 3. Evolución del agua corporal total y masa grasa por BIA 
Caso clínico 2: Mujer de 59 años con insuficiencia renal crónica secundaria a pielonefritis crónica en diálisis peritoneal desde hace unos 6 años y en DPA desde hace casi 5 años (ocho horas y media conectado a cicladora, con 13 litros de intercambio en 5 ciclos con una permanencia de 74 minutos, sistema Baxter). Tras el suplemento encontramos un aumento de $2,4 \mathrm{~kg}$ con respecto al peso de la revisión previa (revisión basal 79,1 kg y a los 2 meses 81,5 $\mathrm{kg}$ ). Asimismo mostraba aumento de la tensión arterial que estaba mal controlada pasando de $147 / 77 \mathrm{mmHg}$ a $150 / 90 \mathrm{mmHg}$, manteniendo el mismo número de hipotensores, con disminución del volumen de ultrafiltración en $24 \mathrm{~h}$ de $350 \mathrm{ml}$ a $100 \mathrm{ml}$ con un discreto aumento del volumen de diuresis de $1300 \mathrm{ml} /$ día pasó a $1400 \mathrm{ml} /$ día. Los niveles de albúmina descendieron de $3,8 \mathrm{~g} / \mathrm{dl}$ a 3,5 g/dl. La exploración física en cuanto a valoración de edemas no cambió presentando edemas $1 / 3$ (en una escala que va desde el cero sin edemas hasta el 3 con edemas importantes) en piernas en el momento basal y siendo igual en el momento final. Con respecto al resto de exploración nutricional encontramos que aumentó la circunferencia de la pantorrilla de $33 \mathrm{~cm}$ a $35 \mathrm{~cm}$ y del brazo de $32,5 \mathrm{~cm}$ a $36,5 \mathrm{~cm}$, y la del muslo bajó un poco de $47,5 \mathrm{~cm}$ a $47 \mathrm{~cm}$ (Tabla I), también aumentó el pliegue graso de la pantorrilla de $19 \mathrm{~mm}$ a $22,5 \mathrm{~mm}$, y descendieron los del muslo de $37 \mathrm{~mm}$ a 29,7 m, el bicipital de $17 \mathrm{~mm}$ a $12 \mathrm{~mm}$ y tricipital de $28 \mathrm{~mm}$ a 20 $\mathrm{mm}$ (Tabla II). Finalmente el análisis de bioimpedancia nos indica que ha aumentado el agua corporal total en 1,6 litros pasando de 31,9 litros a 33,6 litros, con un pequeño aumento de la masa grasa del paciente 38,7 $\mathrm{kg}$ a 39,1 kg (Tabla III).

\section{Discusión}

Ante la elevada prevalencia de malnutrición en pacientes en DP y su asociación con mayor morbi-mortalidad, es necesario poder identificar a los pacientes con riesgo de malnutrición para establecer una adecuada intervención nutricional.

La finalidad de la valoración del estado nutricional es identificar a aquellos sujetos que se encuentren en riesgo nutricional, es decir, a aquéllos en los que existe probabilidad de que su situación nutricional se deteriore. En ellos la intervención nutricional tendrá mayor probabilidad de éxito y buscará evitar el deterioro progresivo y permitir su recuperación, lo cual redundará en una reducción de los riesgos asociados a la malnutrición.
Se han aplicado diversos métodos para evaluar el estado nutricional en pacientes en diálisis no existiendo claramente una técnica "gold standard". Las medidas antropométricas y las determinaciones de laboratorio son las más extendidas. Cuando se aplican a pacientes con insuficiencia renal, la prevalencia de malnutrición que dan es muy variada como se observa en un estudio realizado en pacientes en hemodiálisis ${ }^{9}$. Además algunas de ellas plantean problemas en su aplicación en los pacientes en diálisis por sus especiales características.

El peso corporal es una medida de evaluación nutricional básica y fácilmente disponible. Refleja la suma del compartimento muscular y graso. En pacientes en diálisis presenta cierta variabilidad en el tiempo y es frecuente observar ascensos y descensos del peso de manera periódica o estacional, aunque sean pacientes estables.

La hipoalbuminemia, es el parámetro utilizado tradicionalmente en la evaluación nutricional, ha pasado a considerarse hoy como un parámetro más reflejo de la inflamación que del verdadero estado nutricional de un sujeto. Sus niveles en sangre se ven rápida y fácilmente influenciados por los cambios inflamatorios y del estado de hidratación lo que obliga a buscar otros métodos de valoración nutricional. En este sentido disponemos del análisis de bioimpedancia eléctrica (BIA) que permite valorar la composición corporal de igual manera que otros métodos, así la variabilidad intraindividual para determinar la grasa corporal con bioimpedancia (3,3$3,7 \%$ ) es similar a la variabilidad de la medida del pliegue cutáneo del tríceps $(3,3 \%, p>0,05)^{10}$.

De esto se deduce que aunque existe una elevada prevalencia de malnutrición en pacientes en diálisis peritoneal no disponemos aún de un método único de fácil aplicación y de adecuada fiabilidad para valorar el estado nutricional siendo esta dificultad incrementada por las propias características de los pacientes en diálisis peritoneal como escasa ingesta alimentaria por molestias gastrointestinales por presentar un abdomen Ileno de líquido y sensación de saciedad, la pérdida de nutrientes a través de la propia diálisis peritoneal, los procesos intercurrentes como peritonitis, el estado de expansión de volumen per sé de la técnica, etc.

En este sentido hemos presentado dos casos de pacientes que tomaban un suplemento nutricional y que ambos han ganado peso y que tras la combinación 
de varios métodos observamos que dicho cambio de peso no tiene igual interpretación. De tal forma que en el primer caso clínico se trata de un paciente que tomaba un suplemento nutricional y en la siguiente revisión ganó peso sin apenas encontrar cambios en volumen de ultrafiltración, diuresis y tensión arterial, en el que además ha mejorado nutricionalmente con aumento de los niveles de albúmina y de las medidas antropométricas y la masa grasa sin aumentar su estado de hidratación corporal calculado con BIA. Sin embargo en el segundo caso que también tomaba un suplemento nutricional y también ganó peso, pero con una interpretación diferente de tal forma que disminuyó el volumen de ultrafiltración total, le aumentó la tensión arterial, le bajo la albúmina, no cambió la masa grasa y aumentó el agua corporal total calculado por BIA. Aunque mejoraran algunos parámetros nutricionales, sin la combinación de varios métodos sería difícil interpretar este aumento de peso.

Esta diferente interpretación del aumento de peso es importante porque conlleva actitud terapéutica distinta, de tal forma que en el caso uno hay una buena evolución y mejoría del estado nutricional, pero en el caso dos existe un aumento del líquido corporal lo que nos obliga a llevar a cabo una actitud terapéutica para eliminar ese exceso de volumen circulante. Los casos que hemos mostrado serían casos extremos en los que los datos apuntan claramente hacia un sentido u otro, pero no olvidemos el estado dinámico de los pacientes que están sometidos a cambios continuos con una gran variabilidad interindividual difícil de interpretar por lo que es la experiencia, la adecuada valoración del paciente y la combinación de distintos métodos aplicados conjuntamente por médicos y enfermeros de la consulta lo que nos ayuda en la práctica clínica diaria.

\section{Conclusión}

Existe elevada prevalencia de malnutrición en pacientes en diálisis peritoneal pero no disponemos de un único método fácil y fiable para valorar el estado nutricional. La adecuada interpretación del aumento del peso corporal en un paciente en diálisis peritoneal que ha tomado un suplemento nutricional requiere combinación de varios métodos con un trabajo multidisciplinario en concordancia entre personal médico y de enfermería de la Unidad de Diálisis Peritoneal.

\section{Bibliografía}

1. Piraino B. Recommendations for dietary protein intake in CAPD patients. Adv Perit Dial. 1996; 12:275-279.

2. Li-Tao Cheng, MD, Wen Tang, MD, Tao Wang, D. Strong Association Betwenn Volumen Status and Nutrition Status in Peritoneal Dialysis Patients. Am J Kid Dis. 2005; 45: 891-902.

3. Marcén R., Teruel J.L., de la Cal M.A., Gámez C. and the Spanish Cooperative Study of Nutrition in Hemodialysis. The impact of malnutrition in morbidity and mortality in stable haemodialysis patients. Nephrol Dial Tranplant. 1997. 12: 2324-2331.

4. Fouque $D$, Vennegoor $M$, Ter Wee $P$, Wanner $C$, Basci A, Canaud B. et al. EBPG Guidelines on Nutrition. Nephrol Dial Transplant. 2007;22 (Suppl 2): 45-87.

5. Chen TP, Lee HP, Ghazalli R. Oral nutritional supplement improves nutritional status of CAPD patients. Perit Dial Int. 1998.18 (Supl. 2): S50.

6. Fein PA, Gundumalla G, Jorden A, Matza B, Chattopadhyay J, Avram MM. Usefulness of bioelectrical impedance analysis in monitoring nutrition status and survival of peritoneal dialysis patients. Adv Perit Dial. 2002;18:195-9.

7. Mushnick R, Fein PA, Mittman N, Goel N, Chattopadhyay J, Avram MM. Relationship of bioelectrical impedance parameters to nutrition and survival in peritoneal dialysis patients. Kidney Int Suppl. 2003; 87:53-56.

8. Alaustré A., Sitges A., Jaurrieta E. y Sitges A.: Valoración de los parámetros antropométricos de nuestra población. Med Clín (Barc). 1982. 78: 407-415.

9. Mendías Benítez C, Alonso de Porras L, Barcia García J, Sánchez Oliva JM, Jiménez Quintana E, Lara Ruiz A, Chaín de la Bastida L. Bioimpedancia eléctrica. Diferentes métodos de evaluación del estado nutricional en un centro periférico de hemodiálisis. Rev Soc Esp Enferm Nefrol 2008; 11 (3): 173/177.

10. Pérez S, Parra MD, Blanca E, Martínez d Morentin M, Rodríguez C, Martínez JA. Evaluación de la variabilidad intraindividual de la medida de composición corporal mediante bioimpedancia en voluntarias sanas y su relación con el índice de masa corporal y el pliegue tricipital. Enferm clínica. 2005;15(6):343-7. 